International Journal of Wireless \& Mobile Networks ( IJWMN ), Vol.2, No.3, August 2010

\title{
Algorithm Driven System SELECTION WiTH RECONFIGURABLE SOFTWARE DEFINED RADIO IN MOBILE DEVICES
}

\author{
Preetida Vinayakray Jani \\ Electronics \& Telecommunication Engineering, MPSTME-NMIMS, Mumbai, India \\ preeti_vinayakehotmail.com
}

\begin{abstract}
Emerging global standards for high-speed data, like WiMAX, WiBro, HSDPA and EVDO, presently provide islands of coverage. Such standards require new capabilities from mobile devices. With the coexistence of independent standards, protocols and services it is essential to develop wireless platform that can seamlessly connect to any access networks supported by different standards. Here proposed system architecture supports SDR among multiple communication standards. A logical interface between SDR subsystem and higher level protocol called system reconfiguration manager acts as a logical interface between SDR subsystem and user/application layer. Designed algorithm in SCM enables the proper selection of communication system e.g. OFDM, UMTS etc. and triggers for handover from one communication system to another when user/application specified requirements met by SDR subsystem.
\end{abstract}

\section{KEYWORDS}

Software Defined Radio, Reconfigurability, Seamless Mobility, Wireless Applications

\section{Introduction}

Today multimode mobile terminals are operating in cellular as well as in wireless networks. Also with proliferation of wireless standards in IPTV, radio and mobile communications, compatibility issues have emerged in wireless networks. Inconsistency between wireless standards is causing problems to subscribers, wireless network operators, equipment vendors and such thing is inhibiting deployment of global roaming facilities. Such wireless technologies are stand alone as there is no physical seamless interconnectivity among them. Mobile devices e.g. mobile phone should not be tied to any specific network technology as end user need continuous connectivity while being mobile.

Hence instead of focusing on one standard only it is essential to realize the common denominator among all technologies which can enable continuous connectivity, so that mobile user can roam seamlessly among heterogeneous wireless networking technologies. The required solution should support multiple standards with multibands and multimodes. In the environment where such multiple standard co-exist Software Defined Radio (SDR) plays essential role in realizing seamless switching. By making use of parameterizable hardware modules e.g. Antenna, RF, Modem, Link procedure, INFOSEC etc, it provides flexibility and adaptability required in the converged wireless platforms by implementing the radio functionality as software modules running on a generic platform. It is anticipated that SDR subscriber equipment will include the capability to operate across multiple frequency bands and technologies like OFDM, WCDMA etc. and providing interoperable communication between disparate networks. Thus SDR is capable of transmitting and receiving several different types of wireless systems standards

In SDR based system, goal is to dynamically adapt to any of several air interfaces, such as GSM, GPRS, WCDMA etc, through signal detection and its analysis, and dynamic 
reconfiguration of hardware [7.12], It is similar to today's mobile devices but rather than using multiple RF interfaces, a single reconfigurable RF interface is used. Also SDR is characterized by decoupling between the heterogeneous execution platform of different wireless systems and the applications through firmware/middleware as shown in Figure 1. This heterogeneous execution platform is system on chip which combines microcontroller and baseband signal processing.

SDR attempts to create a flexible platform by using software instead of traditional hardware to perform operations on signals $[2,6,8,9,11]$. Also software approach is quick to compile and load which gives iterative development a much higher cycle rate. The flexibility and ability like dynamic reconfigurability of SDRs' make them prime targets for military and disaster response application. Along with these applicability trend, in addition to many studies on SDR [4], several studies on system selection algorithms of SDR have been discussed $[5,6,7]$ and protocols for inter-system handover have been proposed [3, 10]. In [5], system selection is based on performance of the simple channel model where as in [7] it is based on QoS parameters. Furthermore SDR structures and implementations have been explored in [13, 14, 15] in different ways, but these remain limited to SDR integration with physical layer of system only. In fact, SDR functionality should get integrated with whole system in dynamic manner to optimize mobile host system performance within heterogeneous network.

Therefore in this manuscript an attempt is made to add flexibility to the physical layer and generic platform is built through the design methodology to integrate SDR characteristics with higher layers. With proposed methodology system can analyse the incoming signal and reconfigure the existing wireless application if necessary. Further User/Application level interacts with this SDR system through configuration manager to select proper wireless system. Such interaction will also enable inter-system switching or handover to other wireless standards in order to meet the user/application level requirements.

This manuscript proposes the system architecture where dynamic requirements defined at User/Application level interacts with SDR through system and configuration manager to select proper wireless system. Such interaction will also enable inter-system handover to perform seamless switching from one wireless system to another and there by improving the mobility performance of multihomed host in seamless manner. Besides proposed work also integrates the security component where implementation concept separates the application environment from radio environment and compromise of one will not affect the other.

\section{SOFTWARE DEFINED RADIO: AN OVERVIEW}

As shown in Figure 1[6] SDR consists of front end processing, Radio Frequency (RF) subsystem, Modem or Baseband subsystem. The RF part operates in analog domain which either receives/transmits the $\mathrm{RF}$ signal or down/up converts the signal to/from IF. The functional blocks $\mathrm{ADC} / \mathrm{DAC}$ in modem performs either digital to analog or analog to digital conversion of RF signal. This section also performs Digital Down Conversion (DDC) and Digital Up Conversion (DUP) modulations in receiver and transmitter path respectively. Finally baseband part performs the signal processing functions like Channel coding, Data mapping IFFT/FFT, Channel equalizer, Interleaver/Deinterleaver, Spreader/Despreader, Data mapper/demapper, Encryption/Decryption etc. [3]. 


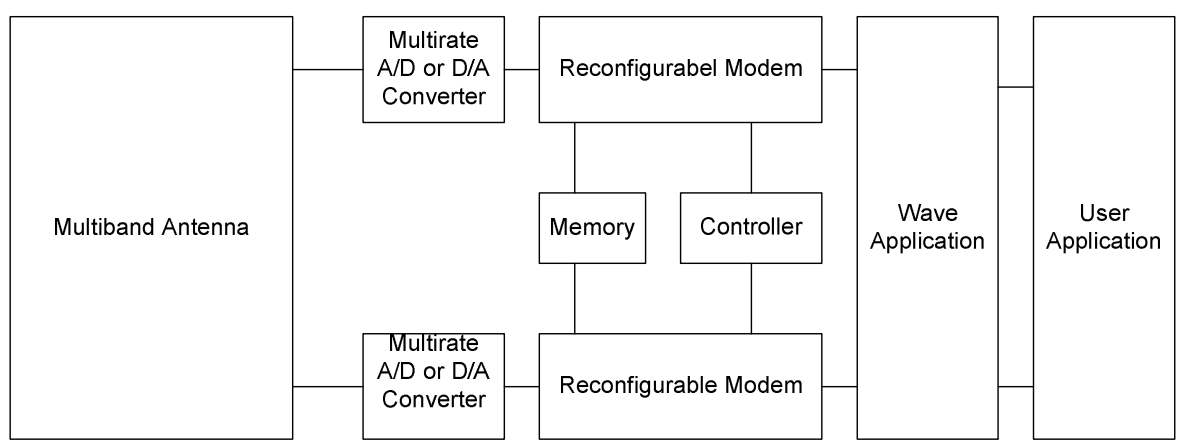

Figure 1 SDR based Transreceiver [6]

A SDR has several sub-systems, and here major sub systems are discussed. Each subsystem contains, hardware, firmware, an operating system and software modules that may be common to more than one application. Application layer is modular, flexible and software specific as it generates many waveform applications. Figure 2 shows layered software architecture of SDR where necessary functional areas are front end processing, information security, information processing and $\mathrm{I} / \mathrm{O}$.

As a part of Infosec subsystem which can facilitate SIM/USIM type hardware and provides user privacy, authentication and information protection. Thus SDR comprises the specific functional block associated with each subsystem as shown in Figure 2. The information transfer is influenced throughout the functional flow within SDR architecture to/from antenna, RF-modem, modem-Infosec and Infosec-message processing interface I/O. The SDR device operates by providing control messages through each of the functional blocks as indicated by control function in platform. As an example the frequency rate which a wireless signal is generated is determined by frequency generation in RF function. Through the control block, SDR device would allow this frequency to be changed to accommodate different operating environment. Such phenomenon is very useful where users move between access systems with different operating frequencies.

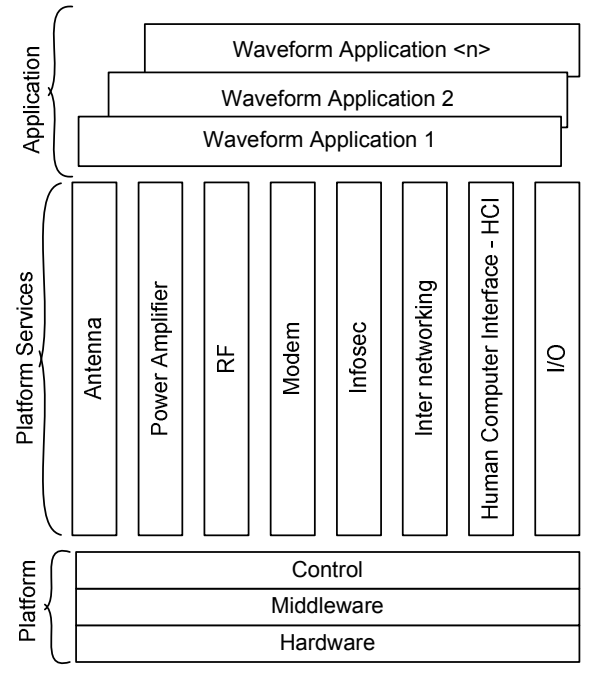

Figure 2 Layered Software architecture in SDR

SDR Reconfigurability is the flexibility based on the ability to add new functions set in baseband processing. For example, because of update of a standard, it can be required to change specific functions in the baseband chain of the running standard because its specifications have changed. This is done by downloading the new versions of the function and then include in the 
processing chain. Eventually proposed system architecture also aims to support flexibility through reconfigurable system manager.

\section{SYSTEM ARCHITECTURE FOR RECONFIGURABILITY}

An integration of SDR as a subsystem and its interaction with main system of mobile terminal shown is Figure 3.

SDR modem driver acts as an interface between SDR modem subsystem and System Configuration Manager (SCM). It also acts as a driver for SDR modem hardware. Once SDR modem device is detected the corresponding driver gets loaded. This driver interacts with the SDR functions to pass command between SCM and SDR functions. Loading of driver in kernel facilitates further baseband signal processing also.

Various waveform drivers loaded/created or unloaded/destroyed when corresponding waveform application is being enabled and unloaded respectively. These drivers are wireless technology specific and modified dynamically when new versions of standard specific derivers become available. SCM is also responsible for loading and unloading of waveform driver modules.

As a part of configuration, SCM stores the configurations and status (i.e. active/deactivate) of each waveform application. It also determines the resources needed by active waveform application and also maintains them. Furthermore SCM also reads the user/application level profile to know the current requirements of the application. If resources corresponding to enabled waveform application are available and waveform characteristics meets the requirements of user level application then such waveform application will get installed and feedback will be given to user application. If resources are not available then it will delete such waveform application and free up all resources reserved for such application. In fact, a loaded waveform application can be activated by SCM only when other SDR subsystem like RF, firmware components is activated and drivers are loaded in the kernel of the system. The SCM also has capability to generate log, error messages or alert signals for functional modules.

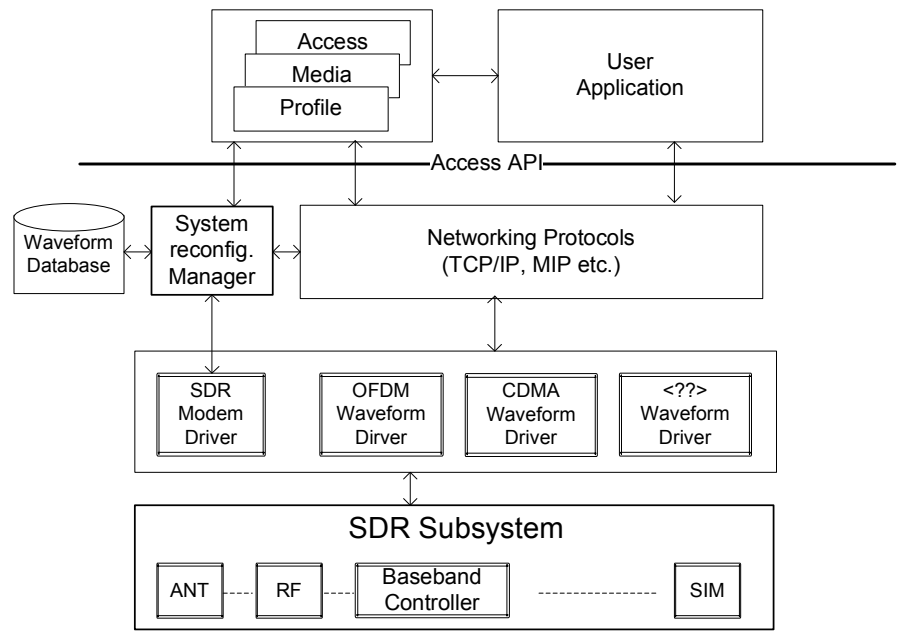

Figure 3 Overall System architecture

Once waveform application is determined by the SDR it triggers the System and Configuration Manager (SCM), a central management function of the whole system architecture of end device. This SCM uses the General Purpose Processor (GPP) resources to perform high-level functions like system management, configuration/reconfiguration management of platform, configuration storage, user interface and so forth. This module performs its functions in conjunction with other platform functions, specifically the SDR executives, user interface and configuration database. The major functions performed by SCM are 
- Initialization and control of modem driver

- Waveform applications management (un)loading, add/delete waveform application from its database, (de)activation

- Trouble shooting or logging support (alert, error, message logging etc.)

\section{System Selection Algorithm}

With proposed algorithm system selection and handoff mechanism from one system to another is determined by system and configuration management. When SDR subsystem analysis's the incoming signal it determines the corresponding waveform application. The installation of this analysed waveform application on mobile device relies on user level application requirements. Such requirements obviously are in terms of quality of service and cost. If user/application requirements are in tune with parameters of analysed waveform application, then system configuration manager installs such waveform application on the system. Also proposed algorithm allows the dynamic selection of waveform application while device is being mobile. Hence proposed system configuration manager should determine the change in user/application requirements as well as change in parameter values of waveform application when device is mobile.

The wireless system selection is a term that can be used for local processing or routing of data through the specific functional modules in a multihomed host. Once the signal received and analysed to identify the corresponding wireless system, it selects the configuration information about the system and reconfigures the waveform application and selects the wireless system. Once the communication on such wireless system is begun, it monitors the channel quality of the unselected wireless system. However the selection of the wireless system also depends on the requirements of the user level application.

In a multihomed host with SDR, the selection of wireless system is dynamic, where mechanism that evaluates actions against the connection related information and decides which wireless system to be used with specific parameters that is discussed below. Hence dynamicity of SDR is not only to select proper wireless system but also such selection should be in consonance with user layer application requirements in terms of quality parameters.

\subsection{Information}

In general, seamless switching between wireless systems is based entirely on network layer information where solution is ad hoc which evaluates the quality of service parameters or security requirements on fly. Moreover as per standards every wireless system has specific security requirements and solution only remains limited to user authentication or specific to one wireless system only. In case of SDR, reconfiguration of wireless applications should be authorized and available quality of service parameters from reconfigured wireless system should be in tune with user level application requirements. In fact, such factors exist below the network layer, thus forcing us to break the level of hierarchy to provide necessary wireless system selection in seamless manner.

- Link layer attributes: In wireless networks the received signal quality and related matrix play important role when deciding which wireless system to use. Though other universal factors must be taken into account, link quality and security is imperative, since it dictates what quality of service demands and policies can be fulfilled and how much of the theoretical bandwidth is available. Moreover, if the link quality is poor, a user with a handheld device might not want to use it at all because of increased power consumption. 
Proposed SDR framework can be further extended to select algorithm that take into account all available information during run time and at the same time minimize the resource consumption and make decision with light computation as possible.

- IP layer information: Although such information is essential for seamless mobility, this manuscript limits its discussion up to here only,

- Network Originated information: A service provider disseminate information about cost, bandwidth and availability. Then transreceiver can then have preferences for connection like maximize bandwidth or minimize cost.

- Users and Applications requirements: Some applications may require certain characteristics from the underlying wireless system. However application should be able to adapt into changing network environment and its own preferences for wireless connections. Apparently this is possible as adaptive applications have certain threshold level requirements in terms of quality of service. Hence application of user level API must allow the delivery of such preferences to the SDR wireless system selection algorithm. Figure 5 shows the application level requirements interaction with analysed and verified signal.

\subsection{Wireless system selection algorithm}

The operation of the wireless system selection mechanism must be dynamic as the signalling information may change at any point of time, i.e. either due to current network unavailability or while being mobile end device comes across totally new wireless access. Being mobile quality of radio signal may change and corresponding signalling waveform application configuration correspond to different wireless system will also change. SCM maintains the database and mechanism to hold and maintain rules for wireless system selection.

The criterion must have some priority defining the order in which specific actions are searched and matched. As shown in Figure 4 such priority should become implicit part of the system as the first criteria relevant action in the list having highest priority where as the default criteria action must always have lowest priority.

The action should include at least one of the following information (i) Unique action ID (ii) a parameter indicating QoS parameters (bit rate, Delay, power consumption etc) or attribute value pair (iii) list of interface types or waveform application characteristics in preferred order (iv) security credentials.

If action is forced then incoming signal is matching to the attribute value pair may not use any other wireless system interface other than the specified in that specific action i.e. Action $\mathrm{X}$ with system A. If action is not forced then all the wireless system listed in that action are unavailable. The next preferred action can be followed by SDR to select available wireless system that is shown in Figure 4. 


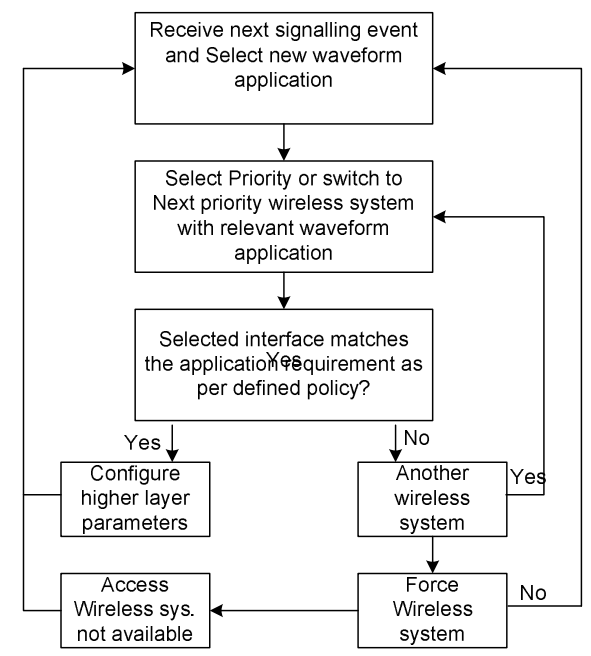

Figure 4 Wireless system selection algorithms

The manuscript focuses on system selection algorithm and mechanism multihomed mobile hosts with SDR. The installation of waveform application to be used for communication relies on user/application defined requirements or criteria. In fact, there are many criteria used to select waveform application such as transmission rate, channel capacity, power consumption and cost. To determine or select which communication system to use the mobile terminal has to prioritize the communication system corresponding to waveform application based on defined criteria. The quality of service parameters are threshold oriented hence communication system availability can be engineered in more adaptive manner but not fixed. Such approach can reduce the number of handovers and thereby improving the user/application level performance.

In addition to this policy also includes signal priority based on security. If preferred security level is available with arrived input signal then only SDR will further process signal to select the wireless system and maintain its security level.

\section{SYSTEM EXECUTION MECHANISM}

The overall system execution where SDR interaction with higher level components and selection of wireless system based on user level application requirements is shown in Figure 5.

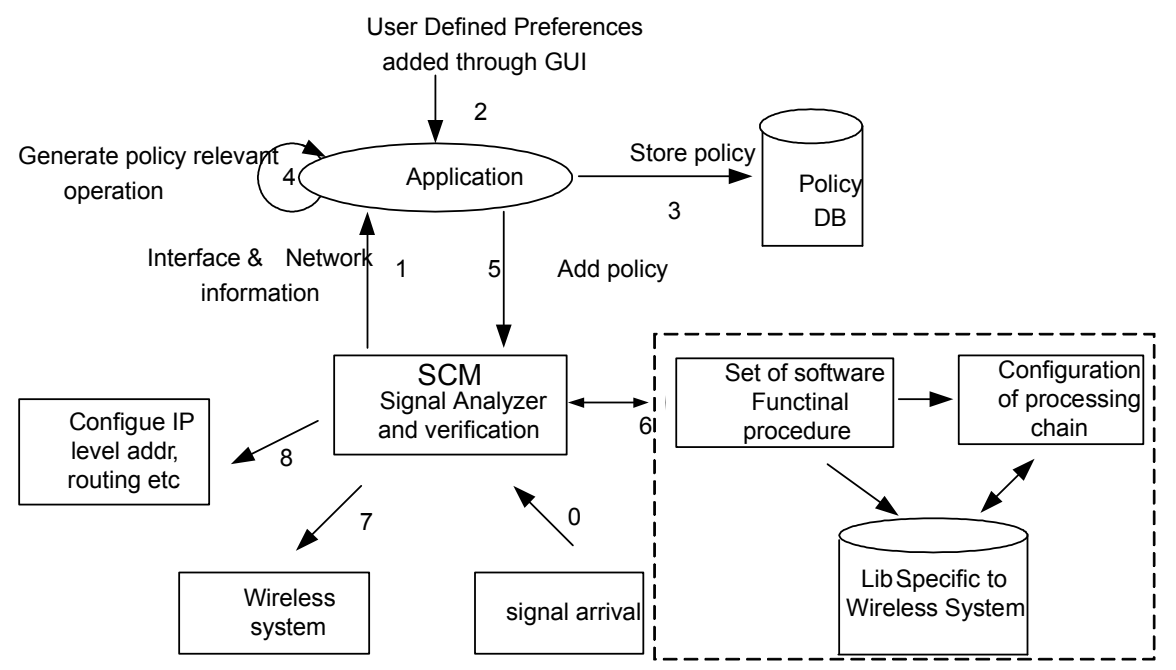

Figure 5 System execution framework 
The system and configuration manager reads user level profile to know the current requirements of user/application. Based on requirements necessary actions are stored in the system selection algorithms defined within SCM. Analyzed signal information is delivered to the management interface which eventually adds the policy to signal analyser. Based on added policy signal analyser interacts with SDR relevant libraries and sets the functional procedure to determine waveform application corresponding to policy. If the outcome of processing chain matches as per added policy the signal analyser selects the wireless system. If such wireless system is available then analyser triggers for configuration of IP based parameters like addressing, routing, mobility etc.

\section{SECURITY CONSIDERATION}

The proposed system architecture already separates the application and radio operation of wireless system so that security compromise of one will not affect the other. Also reconfigurable parameters generated by waveform application will be checked against the security policies before they are processed by functional modules of wireless system. Signal analyser integrated with security monitors checks if reconfiguration is needed for currently ongoing wireless application. Thus separating wireless system radio environment from its radio application supports the modularity, reconfiguration and flexibility goals of designed system architecture in secure manner.

\section{CONCLUSION AND FUTURE WORK}

The proposed system architecture where usage software defined radio is further enhanced to enable mobility management among multiple wireless systems in secure manner. The reusable common functional units reduce complexity of the system and simultaneously support multiple wireless standards. Also reconfiguration and flexibility of system supports integration of new upcoming functional units on fly. A wireless system selection algorithm for SDR based mobile host system is proposed. This proposed algorithm enables system to perform inter-system handover when mobile host is moving across heterogeneous networks. The preliminary results have been encouraging hence next step is to integrate the proposed approach in real-time testbed.

\section{REFERENCES}

[1] Harju, I. Nurmi, J, (2005) “A Programmable Baseband Receiver Platform for WCDMA/ODDM Mobile Terminal”, Proc. of IEEE Wireless Communications and Networking Conference, Vol.1, pp 33-38.

[2] I. Zhigand et al., (2003) “A Multi standard SDR Base Band Platform,” Proc. Of 2003 IEEE International Conference on Computer Networks and Mobile Computing, pp.461-464.

[3] J.Mitola, (1995) “The Software Defined Radio," IEEE Communication Magazine, vol. 33, no. 5, pp 26-38.

[4] T. Tandai et al., (2005) "Soft-prioritization based system selection strategy for Software Defined Radio,” IEEE TRANS COMMUNI, VOL.E88-B, NO. 11.

[5] F. Zhu and J. McNair, (2004) “Optimization for Vertical handoff Decision Algorithm,” Proc In WCNC 2004, Vol. 2, pp.867-872.

[6] T. Shono et al., (2001) "Proposal for System Diversity on Software Defined Radio," IEICE. TRNAS FUNDAMENTALS, vol. E84-A, no. 9.

[7] M. Komara, (2004) C Architecture Ideally Suited for Evolving 802.16 WiMAX,” Published by AirNet Communications, SDR forum Exhibition 
[8] Y Lieng et al., (2005) "Reconfigurable Signal Processing and Hardware Architecture for Broadband Wireless Communications", EURASIP Journal on Wireless Communications and Networking, Vol. 3, pp 323-332

[9] J. Helmshmidt et al., (2003) "Reconfigurable signal processing in wireless terminals [mobile applications]", In proc. Design, Automation and Test in Europe Conference and Exhibition (DATE'03), pp. 244-249, Munich, Germany.

[10] H. Bing, C. He and L. Jiang, (2003) "Performance Analysis of Vertical Handover in UMTSWLAN Integrated Network”, Proc. in PIMRC, Vol. 1, pp. 187-191.

[11] M. Anwar, S. Virtanen and J. Isoaho, (2008)@ A software defined approach for common baseband processing", Journals of System Architecture 54, pp. 769-786.

[12] Z. Wong, A. Erdogan and T. Arslan, (2009) "A SDR Platform for Mobile Wi-Fi/3G UMTS system on a Dynamic Reconfigurable Architecture", $17^{\text {th }}$ European Signal Processing Conference (EUSIPCO 2009), Glasgow, Scotland, pp. 2678-2682.

[13] K. -C. Chen and S. -T. Wu, (2001) "Programmable multiuser Synchronization for OFDMCDMA”, IEEE Communication Magazine, vol. 2, no.11, pp. 76-82.

[14] I. P. Seskar and N. B. Mandayam, (1999) "A Software radio architecture for linear multiuser detection”, IEEE J. Selected Areas of Communication, vol. 17, no.5, pp. 814-823.

[15] J. Helmschmidt et. al., (2003) "Reconfigurable signal processing in wireless terminals [mobile applications]", in Proc .Design, Automation and Test in Europe Conference and Exhibition (DATE'03), pp. 224-249, Munich, Germany.

\begin{abstract}
Author
Short Biography

Preetida Vinayakray Jani received her $\mathrm{PhD}$ in Telecommunication from University of Technology, Sydney, Australia. She was working as a Senior Research Engineer in Nokia, Finland, until accepting professorship in MPSTME-NMIMS, India in 2010. Her research interest includes Wireless communication and Networking, system concept, Security algorithms, Computer networking and implementation techniques.
\end{abstract}

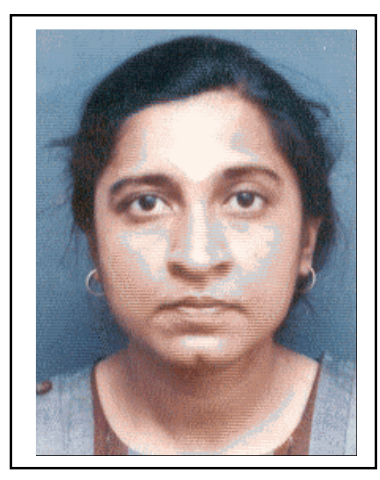

